\title{
Estimating the Characteristics of the Forward Voltage Gain Scattering Parameter of Indoor Power Line Channel Using Only Input Port Measurement
}

\author{
Sujit CHATTERJEE, Rubi BAISHYA, Banty TIRU \\ Dept. of Physics, Gauhati University, India \\ \{sujitc43, rubibaishya89\}@gmail.com,banty_tiru@rediffmail.com
}

Submitted March 8, 2020 / Accepted August 24, 2020

\begin{abstract}
Estimation of the characteristics of power line channel is a pre-requisite for successful implementation of any power line communication system. This paper presents a method to estimate the forward voltage gain scattering parameters $\left(S_{21}\right)$ of an indoor power line using only the input port reflection coefficient $\left(S_{11}\right)$. The measured input parameter with one/two load placed at the output suffices to estimate the $S_{21}$ as far as frequency selectivity is concerned. The positions of notches can be estimated within a limit of error with positive and at times high correlation between the experimental and evaluated $S_{21}$ parameters. The method is validated for a number of practical networks, and also for random channels with different types of loads and cables found in the papers. The procedure can be incorporated in estimating the channel for mitigating the related problems.
\end{abstract}

\section{Keywords}

Power line communication, two port networks, scattering matrices, frequency selectivity

\section{Introduction}

The ubiquitous power lines (PLs) can be used as an alternative or in conjunction with other networking options [1] allowing virtually every line-powered device to become the target of advanced communication services. This has led to Power Line Communication (PLC) in different parts of the PL grid for in-house, access, and control applications requiring both low and high bit rates like smart grids, automation, [1], [2] etc. The problem with PL channel is that it is far from conducive to signal transfer. However, mitigating these would provide cost-effective last-mile last inch solutions penetrating the mass market increasingly.

One of the problems faced by PLC, especially in indoor PLs is the frequency selective nature of the channels [1]. Suitable solutions require an in-depth knowledge or estimation of the channels in terms of their transfer function (TF) [3], scattering parameters ( $S$ parameters) [4], and as to how the signal is scattered, faded, phase-shifted, decayed, noised, etc. before it reaches the receiver. Sufficient knowledge can also be obtained from models and modeling parameters [5-8]. This information can be incorporated in modems for successful mitigation.

The experimental techniques for estimating the PL channel can broadly be divided into the frequency domain (FDA) and the time domain (TDA) approaches. The FDA includes determination of the channel parameters like the $S$ parameters [4], complex frequency response (CFR) [9], access impedance, [10] etc. The CFR can be estimated using the ratio of the voltages at the output and input port, the ratio of the source and the load impedance, measurement of the insertion loss, conversion of $S$ parameters and the use of Scope tool [3], [4], [11], [12]. The TDA includes pilot based methods and blind [13] estimation commonly used in Orthogonal Frequency Division Multiplexing (OFDM). These two methods depend on the transmission and reception of pilots and symbols that are analyzed at the receiver. The use of pilots, however, limits the bandwidth (BW) and the blind estimation is very complex at the receiver. The problem with the cited methods is that it requires two port measurements for estimation and as such difficult to implement. Other techniques are also proposed like mapping the network [14], load frequency mapping [15], adaptive communication [3] and evaluation of the input impedance [16]. The problem with the former three is that it requires a priori knowledge of the topology which is not feasible. The adaptive approach and input impedance method are limited to simulation/emulation only. Another simpler method has been proposed in [17] involving only input port measurements to estimate the TF. This is applied to a twisted copper pair used for the copper access network. Such a technique is however not possible in PLs consisting of a large number of discontinuities caused by heterogeneous components. It has been proposed in [16], that the input impedance (IMP) of two states in a PL channel with different loads at the receiver can give an idea of the TF. However, no validation of the technique exists till date. Suitability of any method must also be accompanied by an in-depth analysis of the extent of validity using experimental results and statistical analysis. A reliable tech- 
nique that uses only input port measurement for two port estimation would simplify the problem in PL channels a great deal. Of all the $S$ parameters, the $S_{21}$ is the most useful [4] as it gives the TF of the network [18].

This paper presents a simple yet effective method to estimate the $S_{21}$ parameter or the forward voltage ratio of a PL channel using the knowledge of only the $S_{11}$ parameter (input port reflection coefficient) for one/two load conditions at the receiver end. Though the exact magnitude may not be feasible, the notches in $S_{21}$ can still be estimated within an error from the frequency dependency. Knowledge of the notches will enable mitigating the problems related to the frequency selectivity (FS). The method represents the PL as a two port network (2PN) and in terms of the $\mathrm{ABCD}$ or chain matrices. This procedure does away with the two port measurements, transmission of signals, and knowledge of the network topology a priori making it simple to implement and also saves the BW. Unlike the IMP method in [16], this technique is realizable in practice, though with some similarity. The one load case further simplifies the implementation and increases the reliability. The method is validated experimentally for a number of test channel and the efficiency found out in terms of the correlation coefficient between the actual and the estimated parameters and the error in determining the frequency of the notches. Statistical analysis is also done using a large ensemble of randomly varying channels that replicate the commonly available networks and cables.

The entire paper is organized as follows: In Sec. 2, the representation of a $2 \mathrm{PN}$ using $S$ parameters and $\mathrm{ABCD}$ matrices is discussed. Section 3 presents the methodology from evaluation to validation for multiple loads. In Sec. 4, the technique is verified experimentally in a number of test channels. Section 5 presents and validates the method using measurements for a single load only. Statistical analysis of random channels follows in the next section. The paper then discusses the results in Sec. 7 and concludes in Sec. 8.

\section{Scattering Parameters and ABCD Matrices of a Two Port Network}

A section of PL network like XY in Fig. 1(a) or even a complex network can be taken as a $2 \mathrm{PN}$ characterized by the $S$ parameters and the ABCD matrix. In the figure, Tx and

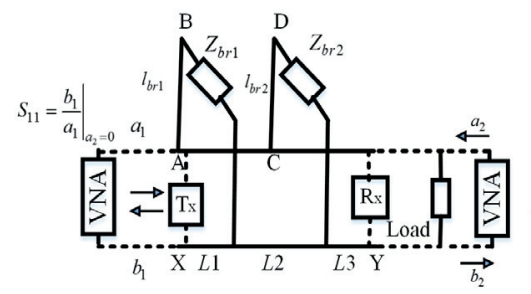

(a)

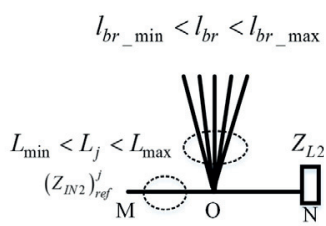

(b)
Fig. 1. (a) A typical power line network showing connection for the proposed method and validation. (b) Reference channel with star branches for estimation using one load.
$\mathrm{Rx}$ are the positions of the transmitter and receiver of PLC devices when used for communication. The four $S$ parameters $\left(S_{11}, S_{12}, S_{21}, S_{22}\right)$ are defined in terms of the incident $\left(a_{1}, a_{2}\right)$ and reflected $\left(b_{1}, b_{2}\right)$ waves at the two ports $\left(b_{1} / a_{1}\right.$, $\left.b_{1} / a_{2}, b_{2} / a_{1}, b_{2} / a_{2}\right)$ with adequate conditions termed as the input port reflection coefficient, reverse voltage gain, forward voltage gain and the output port reflection coefficient respectively [18], [20]. The IMP of a 2PN can be evaluated from the $S_{11}$ parameter and a reference impedance $\left(Z_{\mathrm{r}}\right)$ as given by (1) [18], [20]

$$
Z_{\mathrm{IN}}=Z_{\mathrm{r}}\left(\frac{1+S_{11}}{1-S_{11}}\right) .
$$

The ABCD matrices of a $2 \mathrm{PN}$ are functions of the voltages and current at the two ports and the net matrices are the product of the matrices of the cascaded sections. $Z_{\mathrm{IN}}$ is also related to the net matrices as given by (2) [5]

$$
Z_{\mathrm{IN}}=\frac{A Z_{\mathrm{L}}+B}{C Z_{\mathrm{L}}+D}
$$

Here, $Z_{\mathrm{L}}$ is the load impedance. The relation between the $S$ parameters and ABCD matrices are given by (3)-(6) [21]

$$
\begin{aligned}
& S_{11}=\frac{A Z_{\mathrm{L}}+B-C Z_{\mathrm{S}}^{*} Z_{\mathrm{L}}-D Z_{\mathrm{S}}^{*}}{A Z_{\mathrm{L}}+B+C Z_{\mathrm{S}} Z_{\mathrm{L}}+D Z_{\mathrm{S}}}, \\
& S_{12}=\frac{2(A D-B C) \sqrt{\left|Z_{\mathrm{S}}\right|\left|Z_{\mathrm{L}}\right|}}{A Z_{\mathrm{L}}+B+C Z_{\mathrm{S}} Z_{\mathrm{L}}+D Z_{\mathrm{S}}}, \\
& S_{21}=\frac{2 \sqrt{\left|Z_{\mathrm{S}}\right|\left|Z_{\mathrm{L}}\right|}}{A Z_{\mathrm{L}}+B+C Z_{\mathrm{S}} Z_{\mathrm{L}}+D Z_{\mathrm{S}}}, \\
& S_{22}=\frac{-A Z_{\mathrm{L}}^{*}+B-C Z_{\mathrm{S}} Z_{\mathrm{L}}^{*}+D Z_{\mathrm{S}}}{A Z_{\mathrm{L}}+B+C Z_{\mathrm{S}} Z_{\mathrm{L}}+D Z_{\mathrm{S}}}
\end{aligned}
$$

where $Z_{\mathrm{s}}$ is the source impedance. The ABCD matrices can be evaluated from the reverse formula from the $S$ parameters [21] or theoretically from models of known topologies [5]. For unknown networks, the matrices cannot be obtained from the models because the topology of the PL network is not known. In this paper, the ABCD matrices for unknown networks is obtained by solving equations having known parameters and $S$ parameters evaluated using equations (3)-(6).

\section{Methodology for Multiple Loads}

The flowchart of the method used for evaluation and validation is given in Fig. 2. At first, the $S$ parameters of a 2PN (say XY in Fig. 1(a)) are estimated using a suitable measuring instrument for four loads conditions without the Tx and Rx. The loads $\left(Z_{\mathrm{L} 1}, Z_{\mathrm{L} 2}, Z_{\mathrm{L} 3}, Z_{\mathrm{L} 4}\right)$ are placed at the receiver terminations, one after the other, at the position marked as 'Load' and denoted by $\mathrm{Z}_{\mathrm{L} n}(n=1,2,3,4)$ in general. The topology of the experimental network is considered to be unknown and as such the ABCD matrices from models are also unknown parameters. The $S$ parameters 
obtained are denoted by $\left.S_{11}{ }^{\exp }\right|_{Z \mathrm{~L} n},\left.S_{12}{ }^{\exp }\right|_{Z \mathrm{LL} n},\left.S_{21}{ }^{\exp }\right|_{\text {ZL } n}$ and $\left.S_{22}{ }^{\exp }\right|_{Z \mathrm{~L} n}$. Out of these, only the $\left.S_{11}{ }^{\exp }\right|_{Z \mathrm{~L} n}$ parameter is used in the evaluation stage and the other parameters in the validation stage only. Next, the $\left.S_{11}{ }^{\exp }\right|_{\text {ZL } n}$ parameters are used to evaluate the four IMP of the network using (1) and (7) and represented by $Z_{\mathrm{IN} n}\left(Z_{\mathrm{IN} 1}, Z_{\mathrm{IN} 2}, Z_{\mathrm{IN} 3}, Z_{\mathrm{IN} 4}\right)$ [18], [20].

$$
Z_{\mathrm{IN} n}=Z_{\mathrm{r}}\left(\frac{1+\left.S_{11}^{\exp }\right|_{\mathrm{ZL} n}}{1-\left.S_{11}^{\exp }\right|_{\mathrm{ZL} n}}\right) .
$$

This IMP can also be written in terms of ABCD matrices as given by (2) and (8) [5]

$$
Z_{\mathrm{IN} n}=\frac{A Z_{\mathrm{L} n}+B}{C Z_{\mathrm{L} n}+D} .
$$

Substituting the value of $Z_{\mathrm{IN} n}$ from (7) in (8), four linear equations are obtained as given by (9) with $\mathrm{ABCD}$ as the unknown variables

$$
A Z_{\mathrm{L} n}+B-C Z_{\mathrm{IN} n} Z_{\mathrm{L} n}-D Z_{\mathrm{IN} n}=0 .
$$

The four load conditions keep the matrices of the network XY unchanged. However, being homogeneous, this will give a trivial solution and to make it non-trivial, a constant term $\left(\varepsilon_{n}, n=1,2,3,4\right)$ is added as in (10)

$$
A Z_{\mathrm{L} n}+B-C Z_{\mathrm{IN} n} Z_{\mathrm{L} n}-D Z_{\mathrm{IN} n}=\varepsilon_{n} .
$$

With different values of $\varepsilon_{n}$, the equations can be solved to get a unique solution for the ABCD matrices using mathematical tools. Here, we have used the Gauss's elimination method (GEM) to solve the equations [19]. The ABCD matrices, thus obtained is now applied to re-evaluate back the $S$ parameters using (3)-(6) to give the computed parameters $\left(S_{11}{ }^{\text {comp }}, S_{12}{ }^{\text {comp }}, S_{21}{ }^{\text {comp }}, S_{22}{ }^{\text {comp }}\right)$. While performing these evaluations, the load impedance $Z_{\mathrm{L}}$ is assumed to be $Z_{\mathrm{L} 1}$. As $Z_{\mathrm{L} n}$ are frequency dependent terms, the four equations are constituted at every frequency and the solution obtained at each of these. In the validation stage, the efficiency of the procedure is found out by evaluating the correlation coefficient between the experimental and the computed parameters. The correlation between the magnitude of $\left.S_{11}{ }^{\text {exp }}\right|_{\mathrm{ZL} 1}$ and $S_{11}{ }^{\text {comp }}$ is denoted by $\left(r_{S 11}\right)_{\mathrm{m}}$, between
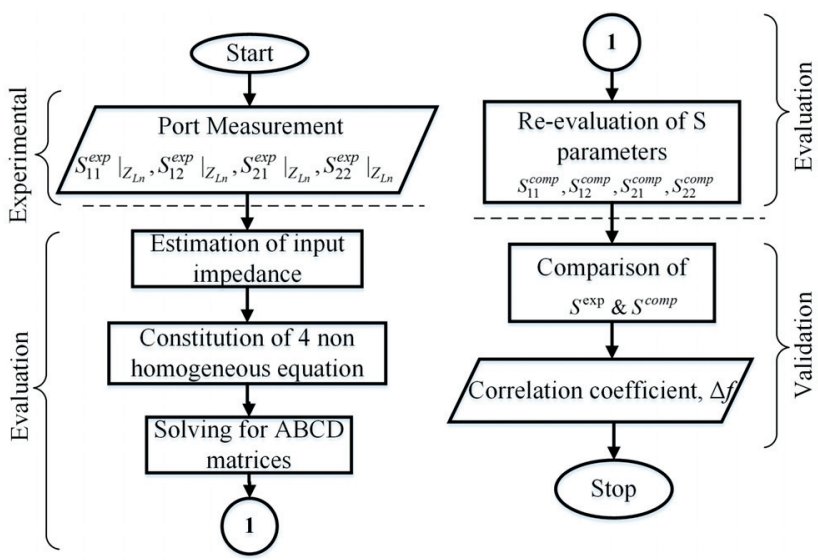

Fig. 2. The flowchart of the method for computation using multiple load.
$\left.S_{12}{ }^{\exp }\right|_{\mathrm{ZL} 1}$ and $S_{12}{ }^{\text {comp }}$ by $\left(r_{S 12}\right)_{\mathrm{m}},\left.S_{21}{ }^{\exp }\right|_{\mathrm{ZL} 1}$ and $S_{21}{ }^{\text {comp }}$ by $\left(r_{S 21}\right)_{\mathrm{m}}$ and $\left.S_{22}{ }^{\exp }\right|_{\mathrm{ZL} 1}$ and $S_{22}{ }^{\text {comp }}$ by $\left(r_{S 22}\right)_{\mathrm{m}}$. Similarly, the correlation between the phase of $\left.S_{11}{ }^{\exp }\right|_{\mathrm{ZL} 1}$ and $S_{11}{ }^{\text {comp }}$ is denoted by $\left(r_{S 11}\right)_{\mathrm{p}}$ and that between $\left.S_{21}{ }^{\exp }\right|_{\mathrm{ZL} 1}$ and $S_{21}{ }^{\text {comp }}$ by $\left(r_{S 21}\right)_{\mathrm{p}}$. Moreover, the frequency of the notches in the magnitude of $\left.S_{21}{ }^{\text {exp }}\right|_{\text {ZL1 }}$ and $S_{21}{ }^{\text {comp }}$ is also found out. The difference in these frequencies $(\Delta f)$ gives the error in estimating the notches using the computed $S_{21}$ parameter. For successful computation, the error $\Delta f$ must be small. For a channel in which there are a number of notches, $\Delta f$ represents the mean error. Though the method has been described for four loads, a three and two load cases are also analyzed to ascertain the condition for successful computation. For this, a modified form of the GEM as described in the next section.

\section{Estimation Using Multiple Loads and Experimental Verification}

The network shown in Fig. 1(a) is used for the analysis and experimental verification. The propagation path XY can have one and/or two branches (AB and $C D)$ as shown in the figure. In practical networks, the branches can be unloaded or loaded $\left(Z_{\mathrm{br} 1}, Z_{\mathrm{br} 2 \ldots}\right)$ conditions and give rise to frequency selective channels due to multipath environment involved. In the network, $L 1=1.25 \mathrm{~m}, L 2=4.50 \mathrm{~m}, L 3=$ $1.10 \mathrm{~m}, l_{\mathrm{br} 1}=3.66 \mathrm{~m}$ and $l_{\mathrm{br} 2}=1.0 \mathrm{~m}$. The primary line parameters of the cables are $L=0.69 \mu \mathrm{H} / \mathrm{m}$ and $C=$ $38 \mathrm{pF} / \mathrm{m}$ with a characteristic impedance of $Z_{0} \sim 135 \Omega$. Four test channels termed as $P L_{1}, P L_{2}, P L_{3}$ and $P L_{4}$ are considered with different configurations as given as follows

1. $P L_{1}$ : One branch $\mathrm{AB}$ (open).

2. $P L_{2}$ : One branch CD (open).

3. $P L_{3}$ : Two branches $\mathrm{AB}$ (open) and $\mathrm{CD}$ (open).

4. $P L_{4}$ : Two branches $\mathrm{AB}$ (short) and $\mathrm{CD}$ (open).

In each of these channels the $S$ parameters are determined using a Vector Network Analyzer (VNA, Keysight Fieldfox N9923A, and $2 \mathrm{MHz}-4 \mathrm{GHz}$ ) for four load conditions $\left(Z_{\mathrm{L} n}=50 \Omega, 100 \Omega, 150 \Omega, 200 \Omega\right)$. The values of the constants $\varepsilon_{n}$ are all taken to be different and given by $\left(10^{-4}, 10^{-3}, 10^{-2}, 10^{-1}\right)$. Two other conditions are also analyzed, using the experimental readings from a three load (say $Z_{\mathrm{L} 1}, Z_{\mathrm{L} 2}, Z_{\mathrm{L} 3}$ ) and a two load (say $Z_{\mathrm{L} 1}, Z_{\mathrm{L} 2}$ ) condition. In the former, three loads are used to constitute the four equations as shown in (11)-(14). In such cases, two equations are constituted having same loads (using $Z_{\mathrm{L} 1}$ ), with different values of $\varepsilon_{1}$ and $\varepsilon_{2}$ and the other two use the remaining two loads $\left(Z_{\mathrm{L} 2}, Z_{\mathrm{L} 3}\right)$ with constants $\varepsilon_{3}$ and $\varepsilon_{4}$.

$$
\begin{aligned}
& A Z_{\mathrm{L} 1}+B-C Z_{\mathrm{IN} 1} Z_{\mathrm{L} 1}-D Z_{\mathrm{IN} 1}=\varepsilon_{1}, \\
& A Z_{\mathrm{L} 1}+B-C Z_{\mathrm{IN} 1} Z_{\mathrm{L} 1}-D Z_{\mathrm{IN} 1}=\varepsilon_{2}, \\
& A Z_{\mathrm{L} 2}+B-C Z_{\mathrm{IN} 2} Z_{\mathrm{L} 2}-D Z_{\mathrm{IN} 2}=\varepsilon_{3}, \\
& A Z_{\mathrm{L} 3}+B-C Z_{\mathrm{IN} 3} Z_{\mathrm{L} 3}-D Z_{\mathrm{IN} 3}=\varepsilon_{4} .
\end{aligned}
$$


The equations can also be written as (15) and (16) in short.

$$
\begin{gathered}
A Z_{\mathrm{L} 1}+B-C Z_{\mathrm{IN} 1} Z_{\mathrm{L} 1}-D Z_{\mathrm{IN} 1}=\varepsilon_{1,2}, \\
A Z_{\mathrm{L} 2,3}+B-C Z_{I N 2,3} Z_{\mathrm{L} 2,3}-D Z_{\mathrm{IN} 2,3}=\varepsilon_{3,4} .
\end{gathered}
$$

In the two load condition, the results of two loads (say $Z_{\mathrm{L} 1}$ and $Z_{\mathrm{L} 2}$ ) are used to constitute the four equations. These can either be (17) and (18) or (19) and (20). In the (17) and (18), two equations have the same load, while at (19) and (20), three equations have the same load with different $\varepsilon_{n}$.

$$
\begin{aligned}
& A Z_{\mathrm{L} 1}+B-C Z_{\mathrm{IN} 1} Z_{\mathrm{L} 1}-D Z_{\mathrm{IN} 1}=\varepsilon_{1,2}, \\
& A Z_{\mathrm{L} 2}+B-C Z_{\mathrm{IN} 2} Z_{\mathrm{L} 2}-D Z_{\mathrm{IN} 2}=\varepsilon_{3,4}, \\
& A Z_{\mathrm{L} 1}+B-C Z_{\mathrm{IN} 1} Z_{\mathrm{L} 1}-D Z_{\mathrm{IN} 1}=\varepsilon_{1,2,3}, \\
& A Z_{\mathrm{L} 2}+B-C Z_{\mathrm{IN} 2} Z_{\mathrm{L} 2}-D Z_{\mathrm{IN} 2}=\varepsilon_{4} .
\end{aligned}
$$

In the three and two load conditions, the system matrix is singular and as such has an infinite number of solutions. An optimum solution is obtained by modifying the GEM keeping in mind that the ultimate aim is to estimate the frequency dependency of parameters and not the exact value. This will give an idea of the notch and hence the FS. In general, the four equations for the three load condition can be written as (21) and (22) for the variables $(x, y, z, w)$

$$
\begin{gathered}
a_{1} x+b_{1} y+c_{1} z+d_{1} w=\varepsilon_{1,2,3}, \\
a_{2} x+b_{2} y+c_{2} z+d_{2} w=\varepsilon_{4} .
\end{gathered}
$$

As this is a singular matrix, the lower triangular matrix cannot be obtained [18]. However, after a number of computations, the matrix form of (23) can be obtained.

$$
\left[\begin{array}{cccc}
a_{1}^{\prime} & b_{1}^{\prime} & c_{1}^{\prime} & d_{1}^{\prime} \\
0 & 0 & 0 & 0 \\
0 & b_{3}^{\prime} & c_{3}^{\prime} & d_{3}^{\prime} \\
0 & 0 & 0 & 0
\end{array}\right]\left[\begin{array}{c}
x \\
y \\
z \\
w
\end{array}\right]=\left[\begin{array}{c}
\varepsilon_{1}^{\prime} \\
\varepsilon_{2}^{\prime} \\
\varepsilon_{3}^{\prime} \\
\varepsilon_{4}^{\prime}
\end{array}\right] .
$$

To get a unique solution of the variables, the diagonal terms are added by an arbitrary small constant $\delta$ that converts the equation to (24):

$$
\left[\begin{array}{cccc}
a_{1}^{\prime}+\delta & b_{1}^{\prime} & c_{1}^{\prime} & d_{1}^{\prime} \\
0 & \delta & 0 & 0 \\
0 & b_{3}^{\prime} & c_{3}^{\prime}+\delta & d_{3}^{\prime} \\
0 & 0 & 0 & \delta
\end{array}\right]\left[\begin{array}{c}
x \\
y \\
z \\
w
\end{array}\right]=\left[\begin{array}{c}
\varepsilon_{1}^{\prime} \\
\varepsilon_{2}^{\prime} \\
\varepsilon_{3}^{\prime} \\
\varepsilon_{4}^{\prime}
\end{array}\right] .
$$

The addition of $\delta$ aids in estimating the frequency dependence of the matrices but not the exact values. The variable $y$ and $w$ is first solved followed by $z$ and $x$. $\delta$ is considered to be $10^{-3}$ in the computation. A similar method is also used for the two load condition.

The efficiency parameters for different load cases are tabulated in Tab. 1 . In the table, $(1,2,3,4)$ signifies that all four loads $\left(Z_{\mathrm{L} 1}, Z_{\mathrm{L} 2}, Z_{\mathrm{L} 3}, Z_{\mathrm{L} 4}\right)$ are used to build up the equations. The set, like $(1,1,2,3)$ that only three loads $\left(Z_{\mathrm{L} 1}, Z_{\mathrm{L} 2}\right.$, $\left.Z_{\mathrm{L} 3}\right)$ are used, $(1,1,1,2)$ and $(1,1,2,2)$ that only two loads $\left(Z_{\mathrm{L} 1}, Z_{\mathrm{L} 2}\right)$ are used and so on. For all the combinations, the correlation $\left(r_{Z}\right)$ between magnitudes of the $Z_{\mathrm{IN}}$ used for computation is also evaluated. For example, for the two load condition say $(1,1,2,2)$ with loads given by $Z_{\mathrm{L} 1}$ and $Z_{\mathrm{L} 2}$, the correlation between the input impedances $Z_{\mathrm{IN} 1}$ and $Z_{\mathrm{IN} 2}$ is evaluated. The correlation is found out by using the expression (25) [22].

$$
r_{z}\left(Z_{\mathrm{IN} 1}, Z_{\mathrm{IN} 2}\right)=\frac{\operatorname{Cov}\left(\left|Z_{\mathrm{IN} 1}\right|\left|Z_{\mathrm{IN} 2}\right|\right)}{\sigma_{\mid \mathrm{ZN} 1} \sigma_{|\mathrm{ZIN} 2|}}
$$

where Cov is the covariance and $\sigma_{|Z I N 1|}$ and $\sigma_{|Z I N 2|}$ are standard deviations of the magnitude of $Z_{\mathrm{IN} 1}$ and $Z_{\mathrm{IN} 2}$ respectively. The frequency resolution in the computation is $100 \mathrm{kHz}$. The computations are considered to be successful for positive values of the correlation coefficients.

From the table, it is seen that computations are mostly successful for $S_{11}$ with positive and high values of $\left(r_{S 11}\right)_{\mathrm{m}}$ and $\left(r_{S 11}\right)_{\mathrm{p}}$. For $S_{21}$, the values of $\left(r_{S 21}\right)_{\mathrm{m}}$ and $\left(r_{S 21}\right)_{\mathrm{p}}$ can be both positive and negative with high and low values. The values of $\left(r_{S 12}\right)_{\mathrm{m}}$ and $\left(r_{S 22}\right)_{\mathrm{m}}$ are mostly negative. The proposed method thus yields successful computations only for $S_{11}$ and $S_{21}$ but not for $S_{12}$ and $S_{22}$. For those cases for which it does give positive results, $\Delta f$ is found out and tabulated. For cases in which the computations are unsuccessful, the $\Delta f$ is stated as 'Not Available' or 'NA' in the table. From the table, it is also seen that an $r_{z}$ ranging from 0.9165 to 0.9999 is obtained. Analyzing the results, the following two conditions need to be satisfied for computation of $S_{21}$.

1. Constitution of four equations from two loads. The first condition is that the four equations must be constituted using only two loads. Though the system matrix of the four load case is non singular, it fails to give the required $S_{21}$ parameter and is noted as 'NA' in the table. The three load case does not give positive results always.

2. The $Z_{\mathrm{L} n}$ for the two loads must have the required correlation. The second condition is that estimation is possible only when the $r_{\mathrm{z}}$ for the two IMPs due to the two loads are not very high $(<0.999)$. The loads chosen to terminate the network must cause an appreciable change in the $Z_{\mathrm{IN}}$. For all combinations of loads in $P L_{4}$, the computation is unsuccessful as $r_{\mathrm{z}}>0.999$ always.

Considering all the successful computations, the correlation for $S_{11}$ and $S_{21}$ can be as high as $\left(r_{S 21}\right)_{\mathrm{m}} \sim 0.9999$ and 0.8679 respectively. The correlation of $\left(r_{S 11}\right)_{\mathrm{p}}$ and $\left(r_{S 21}\right)_{\mathrm{p}}$ is also high. Though $\left(r_{S 21}\right)_{\mathrm{m}}$ can be as low as 0.1347 , the notches can still be estimated with an error of $\Delta f=$ $0.222 \mathrm{MHz}$ (as in $(1,1,1,3)$ of $P L_{2}$ ). Figure 3 and Figure 4 give a plot of the magnitude of $S_{11}$ and $S_{21}$ for $P L_{1}$ for the load conditions $(1,2,3,4)$ and $(1,1,2,2)$, respectively. The figures show that, the computed $S_{21}$ values does not follow 


\begin{tabular}{|c|c|c|c|c|c|c|c|c|c|}
\hline $\begin{array}{c}\text { Test } \\
\text { Channel }\end{array}$ & Loads & $\begin{array}{c}\text { Correlation } \\
\quad\left(r_{z}\right)\end{array}$ & $\begin{array}{c}\text { Correlation } \\
\qquad\left(r_{S 11}\right)_{\mathrm{m}}\end{array}$ & $\begin{array}{c}\text { Correlation } \\
\qquad\left(r_{S 11}\right)_{\mathrm{p}}\end{array}$ & $\begin{array}{c}\text { Correlation } \\
\qquad\left(r_{S 21}\right)_{\mathrm{m}}\end{array}$ & $\begin{array}{c}\text { Correlation } \\
\qquad\left(r_{S 21}\right)_{\mathrm{p}}\end{array}$ & $\begin{array}{c}\text { Correlation } \\
\qquad\left(r_{S 12}\right)_{\mathrm{m}}\end{array}$ & $\begin{array}{c}\text { Correlation } \\
\qquad\left(r_{S 22}\right)_{\mathrm{m}}\end{array}$ & $\begin{array}{c}\text { Mean } \\
\Delta f \\
(\mathrm{MHz})\end{array}$ \\
\hline \multirow{11}{*}{$P L_{1}$} & $(1,2,3,4)$ & -- & 0.9997 & 0.9729 & 0.3322 & 0.3160 & -0.1827 & -0.2321 & NA \\
\hline & $(1,1,2,3)$ & -- & 0.1807 & 0.6945 & 0.7918 & 0.9734 & 0.3812 & -0.1410 & 0.290 \\
\hline & $(1,1,1,2)$ & 0.996 & 0.9997 & 0.9729 & 0.8461 & 0.9832 & -0.2834 & -0.0536 & 0.330 \\
\hline & $(1,1,1,3)$ & 0.996 & 0.9996 & 0.9716 & 0.8679 & 0.9823 & -0.2945 & -0.0322 & 0.185 \\
\hline & $(1,1,1,4)$ & 0.979 & 0.9990 & 0.9716 & 0.8366 & 0.9877 & -0.3030 & -0.0037 & 0.290 \\
\hline & $(1,1,2,2)$ & 0.996 & 0.9803 & 0.9729 & 0.8461 & 0.9769 & -0.2839 & -0.0536 & 0.195 \\
\hline & $(1,1,3,3)$ & 0.989 & 0.9802 & 0.999 & 0.8679 & 0.9796 & -0.2945 & -0.0573 & 0.245 \\
\hline & $(1,1,4,4)$ & 0.979 & 0.9996 & 0.9716 & 0.8366 & 0.9877 & -0.3030 & 0.0037 & 0.47 \\
\hline & $(2,2,3,3)$ & 0.998 & 0.9568 & 0.9327 & 0.8516 & 0.9876 & -0.3627 & 0.0080 & 0.24 \\
\hline & $(2,2,4,4)$ & 0.993 & 0.9647 & 0.9329 & 0.7981 & 0.9918 & -0.2932 & 0.0392 & 0.355 \\
\hline & $(3,3,4,4)$ & 0.998 & 0.9631 & 0.9343 & 0.7139 & 0.9928 & -0.2233 & 0.0817 & 0.265 \\
\hline \multirow{11}{*}{$P L_{2}$} & $(1,2,3,4)$ & ---- & 0.0015 & -0.056 & -0.5329 & 0.9845 & 0.2805 & 0.0848 & NA \\
\hline & $(1,1,2,3)$ & ---- & 0.6439 & 0.6643 & -0.1365 & -0.6537 & -0.1142 & -0.0336 & NA \\
\hline & $(1,1,1,2)$ & 0.9929 & 0.9998 & 0.9912 & 0.1353 & 0.9872 & -0.2594 & -0860 & 1.43 \\
\hline & $(1,1,1,3)$ & 0.9165 & 0.9998 & 0.9912 & 0.1347 & 0.9897 & -0.1291 & 0.5670 & 0.222 \\
\hline & $(1,1,1,4)$ & 0.9516 & 0.9996 & 0.9912 & 0.4890 & 0.9889 & -0.3971 & 0.4798 & 0.115 \\
\hline & $(1,1,2,2)$ & 0.9929 & 0.9998 & 0.9912 & 0.1353 & 0.9872 & -0.1063 & 0.5960 & 1.105 \\
\hline & $(1,1,3,3)$ & 0.9165 & 0.9998 & 0.9912 & 0.1349 & 0.9897 & -0.1291 & 0.5569 & 1.23 \\
\hline & $(1,1,4,4)$ & 0.9516 & 0.9996 & 0.9912 & 0.4890 & 0.9872 & -0.3971 & 0.4797 & 0.852 \\
\hline & $(2,2,3,3)$ & 0.9996 & 0.8783 & 0.6781 & 0.2401 & 0.9437 & 0.0235 & 0.0774 & NA \\
\hline & $(2,2,4,4)$ & 0.9860 & 0.6755 & 0.6447 & 0.6394 & 0.9854 & -0.1768 & -0.3823 & 2.09 \\
\hline & $(3,3,4,4)$ & 0.9862 & 0.0444 & 0.1330 & 0.600 & 0.9865 & -0.1904 & -0.3994 & 1.89 \\
\hline \multirow{11}{*}{$P L_{3}$} & $(1,2,3,4)$ & ---- & 0.9727 & 0.9714 & 0.5270 & -0.1578 & -0.0225 & -0.3575 & NA \\
\hline & $(1,1,2,3)$ & ---- & 0.3824 & 0.3961 & -0.1232 & -0.3090 & -0.0656 & -0.1705 & NA \\
\hline & $(1,1,1,2)$ & 0.9989 & 0.9724 & 0.9714 & 0.5286 & 0.986 & 0.0671 & 0.4584 & 0.672 \\
\hline & $(1,1,1,3)$ & 0.9995 & 0.9726 & 0.9714 & 0.4794 & 0.8308 & 0.0712 & 0.3762 & NA \\
\hline & $(1,1,1,4)$ & 0.9282 & 0.9713 & 0.9714 & 0.4434 & 0.9768 & 0.1123 & 0.1117 & 0.803 \\
\hline & $(1,1,2,2)$ & 0.9989 & 0.9724 & 0.9714 & 0.5286 & 0.9865 & 0.0671 & 0.4584 & 0.667 \\
\hline & $(1,1,3,3)$ & 0.9995 & 0.9726 & 0.9714 & 0.4794 & 0.8308 & 0.0712 & 0.3762 & NA \\
\hline & $(1,1,4,4)$ & 0.9282 & 0.9713 & 0.9714 & 0.4434 & 0.9356 & 0.1123 & 0.1118 & 1.27 \\
\hline & $(2,2,3,3)$ & 0.9997 & 0.1864 & 0.1524 & -0.3028 & -0.5183 & -0.0782 & 0.2755 & NA \\
\hline & $(2,2,4,4)$ & 0.9301 & 0.7459 & 0.9644 & 0.4552 & 0.9628 & -0.1581 & 0.0022 & 1.27 \\
\hline & $(3,3,4,4)$ & 0.9302 & 0.3994 & 0.2859 & 0.4150 & 0.8980 & 0.0692 & 0.0173 & 1.73 \\
\hline \multirow{11}{*}{$P L_{4}$} & $(1,2,3,4)$ & ---- & 0.9999 & 0.9967 & -0.2957 & -0.0813 & -0.1320 & -0.1566 & NA \\
\hline & $(1,1,2,3)$ & ---- & 0.6890 & 0.6636 & -0.4677 & 0.5096 & 0.0114 & 0.1549 & NA \\
\hline & $(1,1,1,2)$ & 0.9997 & 0.9999 & 0.9907 & -0.1826 & -0.6585 & -0.2936 & 0.4569 & NA \\
\hline & $(1,1,1,3)$ & 0.9996 & 0.9999 & 0.9922 & -0.2231 & -0.6585 & -0.3548 & -0.4823 & NA \\
\hline & $(1,1,1,4)$ & 0.9993 & 0.9998 & 0.9992 & -0.2536 & -0.6585 & -0.2605 & -0.4905 & NA \\
\hline & $(1,1,2,2)$ & 0.9997 & 0.9999 & 0.9907 & -0.1826 & -0.6586 & -0.2936 & 0.4569 & NA \\
\hline & $(1,1,3,3)$ & 0.9996 & 0.9999 & 0.9922 & -0.2231 & -0.6586 & -0.3548 & 0.4823 & NA \\
\hline & $(1,1,4,4)$ & 0.9993 & 0.9999 & 0.9922 & -0.2536 & -0.6586 & -0.2605 & 0.4905 & NA \\
\hline & $(2,2,3,3)$ & 0.9999 & 0.9997 & 0.9774 & 0.1390 & -0.6584 & -0.3341 & 0.1618 & NA \\
\hline & $(2,2,4,4)$ & 0.9995 & 0.9996 & 0.9876 & -0.1520 & -0.6578 & -0.2373 & 0.2116 & NA \\
\hline & $(3,3,4,4)$ & 0.9998 & 0.9970 & 0.9975 & -0.0428 & -0.6555 & -0.0241 & 0.1680 & NA \\
\hline
\end{tabular}

NA: Not Available

Tab. 1. Correlation between different parameters for different load condition and the deviation in the frequency of notches.

the experiment in the four load case, but are very similar in the two load case. The former shows no notches and is noted as ' $N A$ ' in the table. However, the latter gives a notch with a mean error $\Delta f=0.33 \mathrm{MHz}$. In the latter, the discontinuity in the phase plot at the notch position is also clearly seen. In the two load case (Fig. 4), the magnitudes of the computed and experimental case may be different, but the frequency dependency (shape) is nearly similar. Figure 5 shows the magnitude of the IMP for $(1,1,2,2)$ for $P L_{1}$ and $P L_{4}$ with an $r_{z}$ value of 0.996 and 0.9997 respectively. As seen from the table, the computation is successful only for the former but not so for the latter, because of high value.

\section{Estimation Using Single Load and Experimental Verification}

It can be concluded from the previous section that computation of $S_{21}$ is successful when there are two channels whose IMP are suitably correlated $\left(r_{\mathrm{Z}}<0.999\right)$. These two channels must be the same except in the value of the terminating load. As such, one can be taken as a reference upon which the computation is carried out. In the two load condition, both the channels are the experimental ones with different values of the load. In this section, the idea of reference is extended to the evaluation of $S_{21}$ from the knowledge of the $S_{11}$ for only one load. As the computa- 
tions require two channels, the experimental is considered as the first channel and a simulated one as the other. The simulated channel is termed to here as the reference channel (RC).
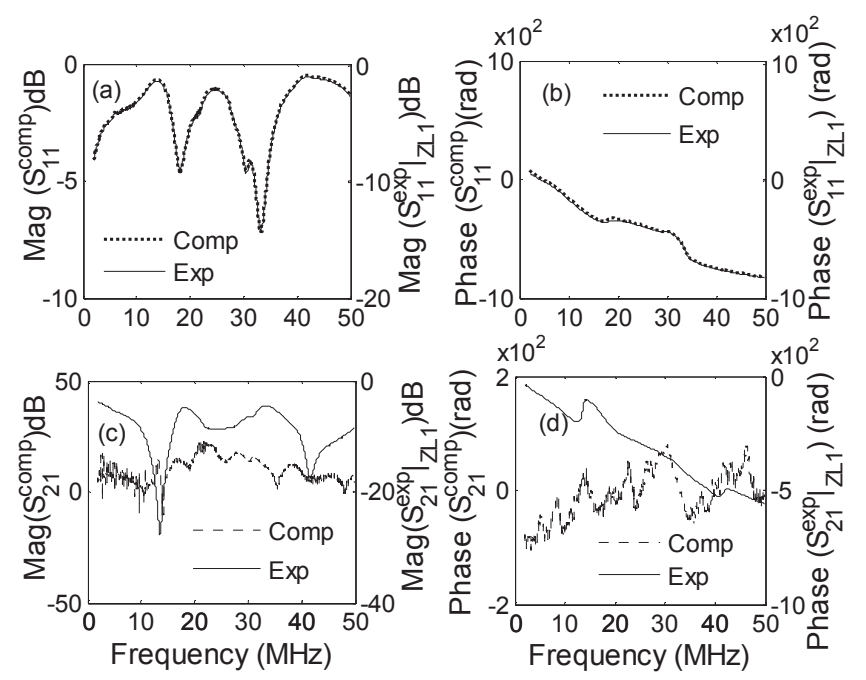

Fig. 3. The magnitude and phase plot of both computed (Comp) and experimental (Exp) values of $S_{11}$ and $S_{21}$ for $P L_{1}$ in the case $(1,2,3,4)$ showing unsuccessful computation of $S_{21}$ but successful $S_{11}$.
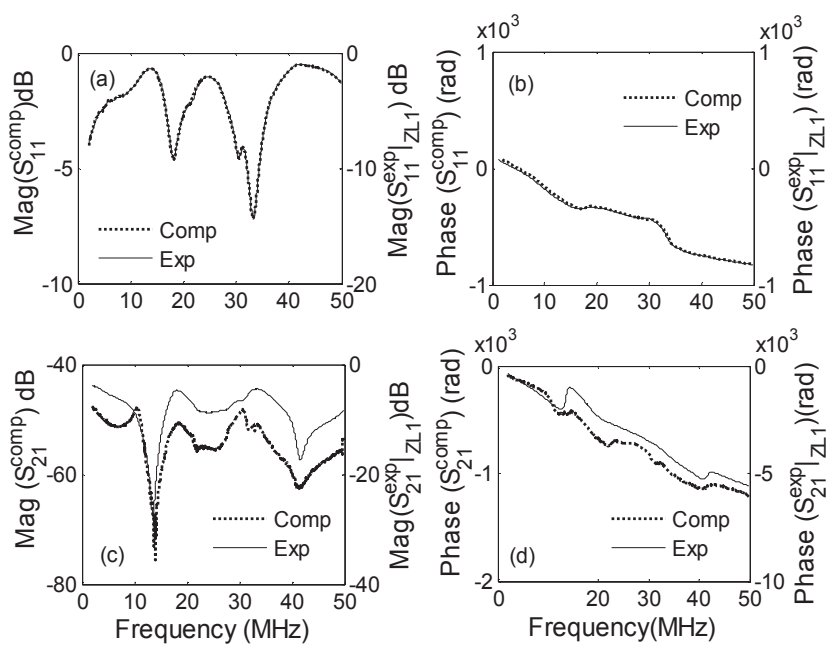

Fig. 4. The magnitude and phase plot both computed (Comp) and experimental (Exp) of $S_{11}$ and $S_{21}$ for $P L_{1}$ in the case $(1,1,2,2)$ showing successful computation of both $S_{11}$ and $S_{21}$.
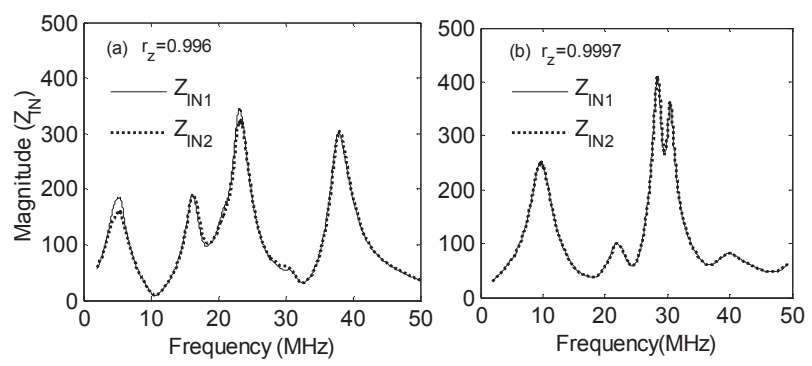

Fig. 5. The magnitude of the input impedance $Z_{\mathrm{IN} 1}$ and $Z_{\mathrm{IN} 2}$ for the case $(1,1,2,2)$ for (a) $P L_{1}$ and (b) $P L_{4}$ showing low correlation and high correlation respectively.

\subsection{The Reference Channels}

The RC is based on a number of observations. Firstly, the frequency of the notches in the TF of a PL section is dependent on the nature of the branched taps but not on the position in the propagation path [5]. The attenuation of the notches is, however, dependent on the latter [5]. Secondly, the notches due to multiple branches in a PL are at all the positions as for the branches considered individually [15]. Thirdly, a star branch as shown in Fig. 1(b) having branches of various lengths has notches at various frequencies. Fourthly, when a simulated network is taken as a $\mathrm{RC}$, the computation with the experimental reading will give a notch in the correct position only if the former has a close similarity with the experimental (Section 4). In such a case, the length of the branch and the position in the propagation path should match that of the experimental one. This variability is, however, not known a priori for practical PL networks.

As the length of the branches that represents the experimental is not known, a RC shown in Fig. 1(b) is simulated. The network has star branches in open circuited conditions with lengths between a maximum $\left(l_{\text {br_max }}\right)$ and minimum $\left(l_{\text {br_min }}\right)$ and a consecutive difference of $\Delta l$. The TF of such a network will have notches at various frequencies, out of which only a few will correlate with the experimental. Also, as the star branch can be placed in any position in $\mathrm{MN}$, a number of RCs need to be considered with differing length of MO. The smallest and the largest value of MO can be $L_{\min }$ and $L_{\max }$ respectively, with a consecutive difference of $\Delta L$. As every variant of a $\mathrm{RC}$ is known, the net ABCD matrices can be evaluated theoretically using $T_{\text {net }}=T_{j} X T_{\text {star }} X T$ where $T_{j}, T_{\text {star }}$ and $T$ are the ABCD matrices of the transmission line (TL) represented by MO, the star branch and ON respectively. The IMP of the RC can then be obtained using (8) to constitute the required equations. For a single unknown experimental network, the computation is carried with $N$ number of RCs with different lengths of MO.

\subsection{Methodology Using the Reference Channels}

The flowchart of the method involving the RCs is given in Fig. 6. It consists of three parts: the experimental evaluation of the $\left.S_{11}{ }^{\exp }\right|_{\mathrm{ZL} 1}$, the formation of a RC and the computation of $S_{21}$ and validation. In the first step, the experimental value of the $S_{11}$ parameter is evaluated using a VNA for the load $Z_{\mathrm{L} 1}$ at the receiver termination. This is represented by $\left.S_{11}{ }^{\exp }\right|_{\mathrm{ZL} 1}$ and used to evaluate the IMP of the network $\left(Z_{\mathrm{IN} 1}\right)_{\exp }$ from (7). The IMP constitutes two equations (26) with constants $\varepsilon_{1}$ and $\varepsilon_{2}$. These are represented by $f\left\{\left(Z_{\mathrm{IN} 1}\right)_{\exp }, Z_{\mathrm{L} 1}\right\}=\varepsilon_{1,2}$ in the flowchart.

$$
A Z_{\mathrm{L} 1}+B-C\left(Z_{\mathrm{IN} 1}\right)_{\exp } Z_{\mathrm{L} 1}-D\left(Z_{\mathrm{IN} 1}\right)_{\exp }=\varepsilon_{1,2} .
$$

Next, a RC is simulated with the star branch placed in an arbitrary position with the length of MO given by $L_{j}$. 


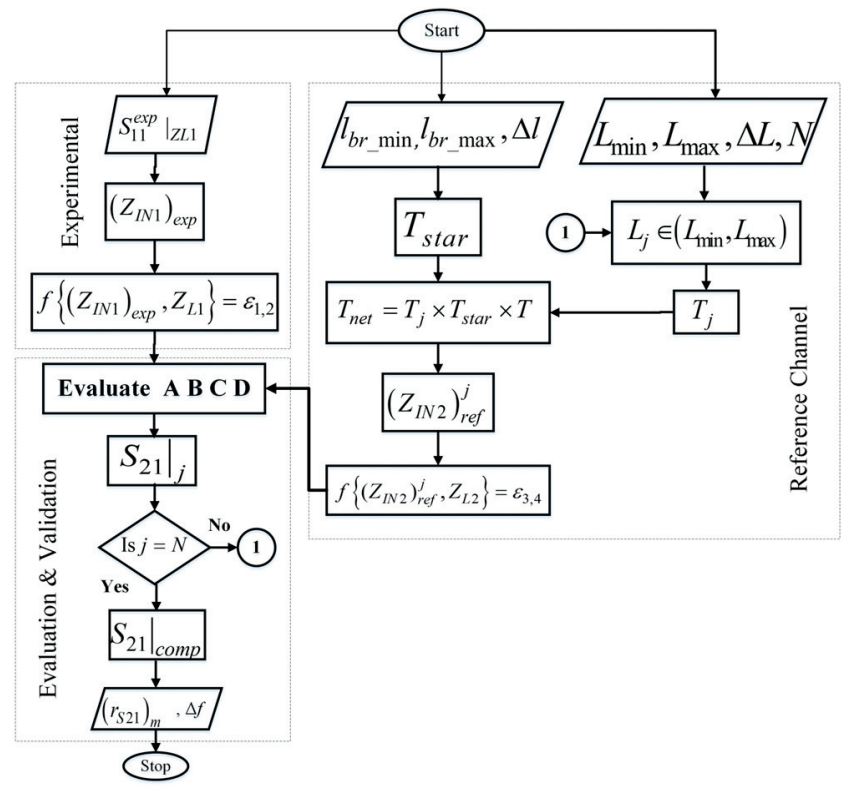

Fig. 6. The flowchart for computation using single load.

The line parameters of the cable in the simulation must be the same as the experimental network. The IMP $\left.\left(Z_{\mathrm{IN} 2}\right)\right|_{\text {ref }} ^{j}$ of this network is obtained from the modeled ABCD matrices and constitutes the third and fourth equations as given by (27)

$$
A Z_{\mathrm{L} 2}+B-C\left(Z_{\mathrm{IN} 2}\right)_{\mathrm{ref}}^{j} Z_{\mathrm{L} 2}-D\left(Z_{\mathrm{IN} 2}\right)_{\mathrm{ref}}^{j}=\varepsilon_{3,4} .
$$

The load at the termination in the $\mathrm{RC}$ is taken to be $Z_{\mathrm{L} 2}$ and the equations are represented by $f\left\{\left(Z_{\mathrm{IN} 2}\right)_{\text {ref }}^{j}, Z_{\mathrm{L} 2}\right\}=$ $\varepsilon_{3,4}$ in Fig. 6. Equations (26) and (27) are analogous to (17) and (18), respectively. As before, the $S_{21}$ parameter is computed solving the four equations using the modified GEM and is denoted by $\left(\left.S_{21}\right|_{j}\right)$ in the flowchart. As the position of the star branch in $\mathrm{MN}$ is not known, such a computation is carried out with $N$ number of RCs with different lengths of MO. If $j<N$, the next value of the length $L_{j+1}$ is taken and the same procedure is followed to give the computed $S_{21}$ parameter. From all the computed $S_{21}$ parameters, the final value is obtained by estimating the minimum at every frequency as given by (28).

$$
\left.S_{21}\right|_{\text {comp }}=\left.\left(S_{21}\right)_{1<j<N}\right|_{\min } .
$$

It must be noted that only for the RC that has a similar topology as the unknown channel is the computation successful. The ABCD solved in such a case will give the required $S_{21}$ parameter. The computed parameters are compared with the experimental one to give the efficiency parameters.

\subsection{Validation of the Single Load Method}

The single load method is validated for all the test channels $P L_{1}-P L_{4}$. For this, the experimental reading of the $S_{11}$ parameter for only one load is taken and computed with $N$ number of RCs. Figure 7(a) shows all the computations when $L_{j}$ is changed between the two extremities in the
RC for $P L_{1}$ and denoted by 'All computation'. It is seen from the figure that a large number of notches concentrate on the actual position and the minimum at every frequency gives the final 'computed' value. Figure 7(b) shows the normalized plot of the experimental and final computed parameter. It is seen that the a high $\left(r_{S 21}\right)_{\mathrm{m}}$ of 0.532 and a mean $\Delta f$ of $0.33 \mathrm{MHz}$ is obtained. Figure 8 shows the results from the other test channels as well. In all the cases, the computed value shows nearly similar variation as the experimental. Similar computations are performed for channels with branches having frequency dependent loads namely

1. $P L_{5}$ : One branch $\mathrm{AB}$ having a capacitive load $\left(10^{3} \mu \mathrm{F}\right)$.

2. $P L_{6}$ : One branch $C D$ having an inductive load $(90 \mu \mathrm{H})$.

3. $P L_{7}$ : One branch $\mathrm{AB}$ with parallel RLC load $(10 \mathrm{k} \Omega$, $90 \mu \mathrm{H}, 5 \mathrm{pF})$.

Figure 9 also shows the results for these test channels. Table 2 notes the efficiency parameters for all the channels

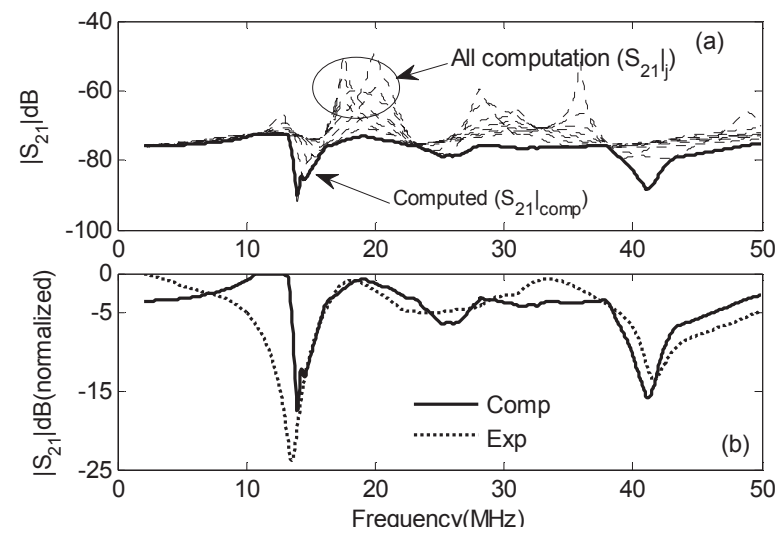

Fig. 7. The magnitude of $S_{21}$ for estimation using one load for the test channel $P L_{1}$ shown for (a) all computations using all the reference channels considered and the computed using the minimum at every frequency; (b) normalized experimental (Exp) and the computed (Comp) values.
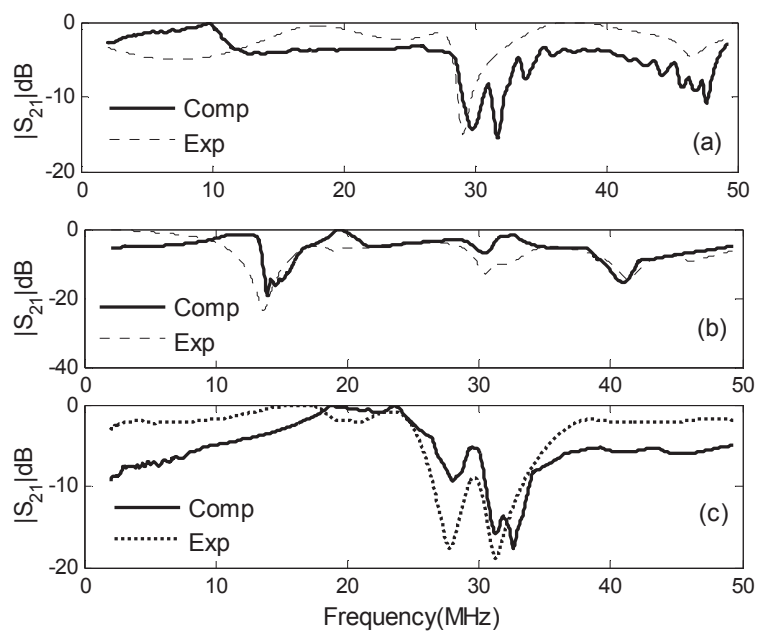

Fig. 8. The experimental (Exp) and computed (Comp) magnitude of $S_{21}$ (normalized) for the test channels: (a) $P L_{2}$, (b) $P L_{3}$ and (c) $P L_{4}$ when evaluated using one load technique. 


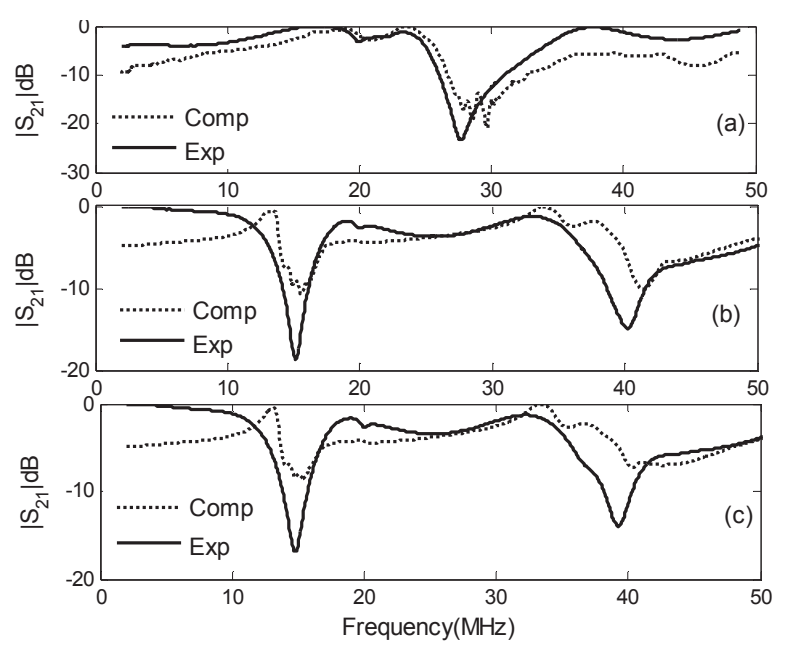

Fig. 9. The experimental (Exp) and computed (Comp) magnitude of $S_{21}$ (normalized) for the test channels: (a) $P L_{5}$, (b) $P L_{6}$ and (c) $P L_{7}$ when evaluated using one load technique.

\begin{tabular}{|c|c|c|c|c|c|c|c|}
\hline Parameters & $\boldsymbol{P L}_{\mathbf{1}}$ & $\boldsymbol{P L}_{\mathbf{2}}$ & $\boldsymbol{P L}_{\mathbf{3}}$ & $\boldsymbol{P L}_{\mathbf{4}}$ & $\boldsymbol{P L}_{\mathbf{5}}$ & $\boldsymbol{P L}_{\mathbf{6}}$ & $\boldsymbol{P} \boldsymbol{L}_{7}$ \\
\hline$\left(r_{S 21}\right)_{\mathrm{m}}$ & 0.532 & 0.517 & 0.521 & 0.546 & 0.793 & 0.500 & 0.501 \\
\hline$\Delta f(\mathrm{MHz})$ & 0.330 & 0.50 & 0.367 & 0.147 & 0.840 & 0.980 & 0.255 \\
\hline
\end{tabular}

Tab. 2. Correlation and mean $\Delta f$ for different test channels in case of evaluation using one load condition.

considered. A high correlation and low deviation $(\Delta f<1 \mathrm{MHz}$ ) is obtained showing that the proposed method is valid both loaded and unloaded branches in the network.

\section{Statistical Analysis of the Method}

In order to perform a statistical analysis of the validity of the method and also for different cables, random channels are constituted and verified using the flowchart shown in Fig. 10. The channels are constituted for a multi-tap, multi-branch system with all the variability like number of branches, lengths of branches and TL, type of loads etc. selected randomly. The loads at the branches are taken to be capacitive $(\mathrm{C})$, inductive (L), resistive (R) or RLC circuit (parallel) loads. The values of the loads are within the ranges given by $C \in(0.1,0.47)_{\text {min,max }} \mu \mathrm{F}$ [13], $L \in(0.1$, $100)_{\min , \max } \mathrm{mH}$ [13] and $R \in\left(0,10^{5}\right)_{\min , \max } \Omega$ [13] given in the papers. For RLC loads, the resistance at resonance $R$, the quality factor $Q$, the angular frequency $w_{\mathrm{r}}$ and the frequency at resonance $f_{\mathrm{r}}$ vary within limits $R \in(200,1800) \Omega$, $f_{\mathrm{r}} \in(2,28) \mathrm{MHz}$ and $Q \in(5,25)$ [13]. These random channels are the networks whose $S_{21}$ parameters need to be computed from single port measurement. The channels are taken analogous to experimental unknown channels though in simulation only as practical networks are not available. For each random channel, two quantities are evaluated using the net $\mathrm{ABCD}$ matrices. The net $\mathrm{ABCD}$ matrices are given by $T_{\text {net }}=T_{1} X T_{2} X \ldots$. where $T_{1}$ and $T_{2}$ are the matrices of the cascaded sections and so on. The first is the $S_{21}$ parameter from (5) and the second is the $Z_{\mathrm{IN}}$ from (2). The $S_{21}$ parameter obtained is taken as the 'actual' as if it were from the experimental readings. The two quantities are denoted by $\left.S_{21}\right|_{\text {actual }}$ and $\left(Z_{I N 1}\right)_{\text {random, respectively. It is }}$ required to evaluate $\left.S_{21}\right|_{\text {actual }}$ using only the information about $\left(Z_{\mathrm{IN} 1}\right)_{\text {random. }}$ This IMP is used to constitute the two equations as given by (29). This equation is similar to (26) though in simulation only and is written as $f\left\{\left(Z_{\mathrm{IN} 1}\right)_{\text {random }}, Z_{\mathrm{L} 1}\right\}=\varepsilon_{1,2}$ in the flowchart. As before, the other two equations (30) are constituted using the RC and denoted by $f\left\{\left(Z_{\mathrm{IN} 2}\right)_{\text {ref },}^{j} Z_{\mathrm{L} 2}\right\}=\varepsilon_{3}, 4$

$$
\begin{gathered}
A Z_{\mathrm{L} 1}+B-C\left(Z_{\mathrm{IN} 1}\right)_{\text {random }} Z_{\mathrm{L} 1}-D\left(Z_{\mathrm{IN} 1}\right)_{\text {random }}=\varepsilon_{1,2}, \\
A Z_{\mathrm{L} 2}+B-C\left(Z_{\mathrm{IN} 2}\right)_{\mathrm{ref}}^{j} Z_{\mathrm{L} 2}-D\left(Z_{\mathrm{IN} 2}\right)_{\text {ref }}^{j}=\varepsilon_{3,4} .
\end{gathered}
$$

Solving these four equations using the modified GEM method, the $S_{21}$ parameter is computed for multiple RCs and $\left.S_{21}\right|_{\text {comp }}$ evaluated using (28). This is done for 250 numbers of random channels. Figure 11 shows the normalized $\left.S_{21}\right|_{\text {comp }}$ and $\left.S_{21}\right|_{\text {actual }}$ for one of the channels. It is seen that a high correlation of $\left(r_{S 21}\right)_{\mathrm{m}} \sim 0.81$ and a mean $\Delta f$ of $0.1 \mathrm{MHz}$ is obtained. Similar analysis is done for cables referred in papers [9] (Cables $\mathrm{C} 1-\mathrm{C} 6$ ). Table 3 gives the mean $\left(r_{S 21}\right)_{\mathrm{m}}$ and mean $\Delta f$ of the channels generated. It is seen that for all the cables, $\left(r_{S 21}\right)_{\mathrm{m}}$ is large and $\Delta f$ is small.

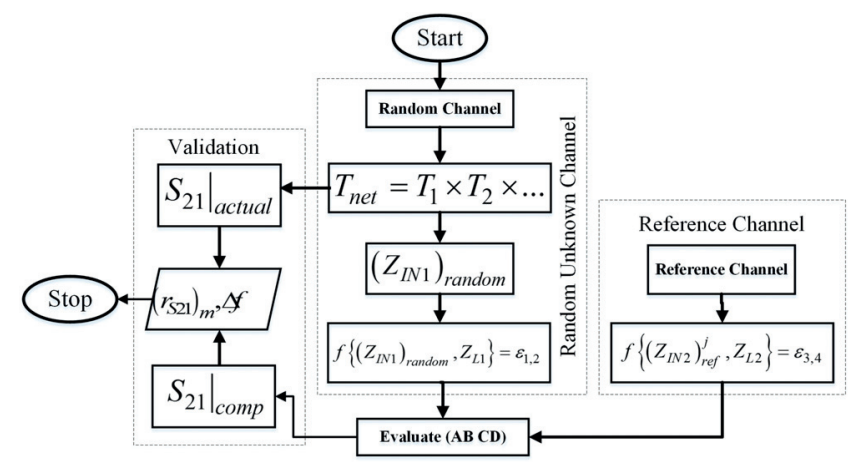

Fig. 10. The flowchart of the method for statistical analysis of random channels.

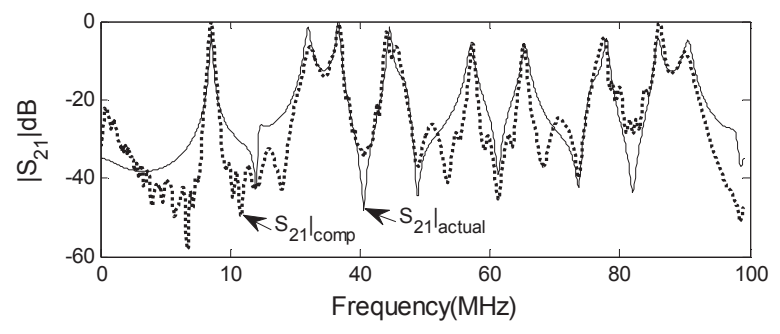

Fig. 11. The normalized $S_{21}$ parameter (actual and computed) for a random channel generated.

\begin{tabular}{|c|c|c|c|c|c|}
\hline Cable[9] & $\boldsymbol{L}(\boldsymbol{\mu H} / \mathbf{m})$ & $\boldsymbol{C}(\mathbf{p F} / \mathbf{m})$ & $\boldsymbol{Z}_{\mathbf{0}}(\boldsymbol{\Omega})$ & $\left(r_{S 21}\right)_{\mathrm{m}}$ & $\boldsymbol{\Delta f}(\mathbf{M H z})$ \\
\hline $\mathrm{C} 1^{*}$ & 0.69 & 38 & 135 & 0.637 & 0.261 \\
\hline $\mathrm{C} 2$ & 1.08 & 15 & 270 & 0.681 & 0.452 \\
\hline $\mathrm{C} 3$ & 0.96 & 17.5 & 234 & 0.623 & 0.293 \\
\hline $\mathrm{C} 4$ & 0.87 & 20 & 209 & 0.613 & 0.212 \\
\hline C5 & 0.78 & 25 & 178 & 0.643 & 0.268 \\
\hline C6 & 0.68 & 33 & 43 & 0.657 & 0.207 \\
\hline
\end{tabular}

*Present Cable

Tab. 3. Mean correlation and mean $\Delta f$ for different cables. 


\section{Results and Discussion}

The proposed method enables the notches in the $S_{21}$ parameters to be computed from $S_{11}$ and is verified for a number of channels and cables. Though the correlation $\left(r_{S 21}\right)_{\mathrm{m}}$ is low at times, and the overall magnitude differs, the position of the notches can still be determined within a limit of error giving an idea of the FS. The $S_{12}$ and $S_{22}$ parameters cannot be evaluated using this method, but it suffices to know the $S_{21}$ as this is related to the TF and of considerable interest for communication. The success of the method is because the position of the notches is independent on the load at the terminations but is primarily dependent on the branches which remain unchanged for different load conditions. The modification of the GEM has enabled the evaluation of the frequency dependency of at least $\mathrm{A}$ and $\mathrm{C}$. Figure 12 shows the plot of the absolute values of the $\mathrm{A}, \mathrm{B}, \mathrm{C}$ and $\mathrm{D}$ evaluated from the experimental $S$ parameters $\left(\mathrm{A}_{\text {exp }}, \mathrm{B}_{\text {exp }}, \mathrm{C}_{\text {exp }}, \mathrm{D}_{\text {exp }}\right)$ and solving the equations $\left(\mathrm{A}_{\text {comp }}, \mathrm{B}_{\text {comp }}, \mathrm{C}_{\text {comp }}, \mathrm{D}_{\text {comp }}\right)$ for the case $(1,1,2,2)$ in $P L_{1}$. The former are obtained from the reverse formulas of $S$ parameters to the ABCD matrices [21]. It is seen that the modification of the GEM has resulted in constant values of $\mathrm{B}_{\text {comp }}$ and $\mathrm{D}_{\text {comp }}$ but the $\mathrm{A}_{\text {comp }}$ and $\mathrm{C}_{\text {comp }}$ follow the experimental counterparts having a high correlation coefficient of 0.7268 and 0.6793 , respectively with them. When the four values are substituted in (5), the frequency dependency of $\mathrm{A}_{\text {comp }}$ and $\mathrm{C}_{\text {comp }}$ enable the frequency dependency of $S_{21}$ to be obtained even when $\mathrm{B}_{\text {comp }}$ and $\mathrm{D}_{\text {comp }}$ have constant values. As the requirement is get an idea of the FS, this approximation suffices. In the computation, the values for $\varepsilon_{n}$ are arbitrary and result only in a change in the magnitude of the computed value of $S_{21}$ keeping the frequency dependency same. The constant $\delta$ also changes the magnitude of the evaluated parameters. Though $\delta=10^{-3}$ in the computation, the use of $\delta=1$, causes the magnitude of the computed $S_{21}$ to be very nearly equal to the experimental value. The two-load condition is successful only if the $r_{z}$ satisfies the required conditions and requires the load be selected carefully. However, the one load condition removes this problem and enables determination of notches even when it is unsuccessful for the former (for example with $P L_{4}$ ). For successful one load computation, it necessitates that $\Delta l$ and $\Delta L$ is as small as possible. As the line parameters $L$ and $C$ are the prerequisites of the star network used as reference, it is required that these parameters be determined as accurately as possible. The method proposed is tested for channels that are time invariant in nature. Considering the case that PL channels are slowly varying channels, a continuous analysis of $S_{11}$ would enable evaluation of the $S_{21}$ parameters for time varying channels also.

\section{Conclusion}

In this paper, a method for estimating the characteristics of a PL channel specifically the FS is presented where a two port parameter can be obtained from a single port
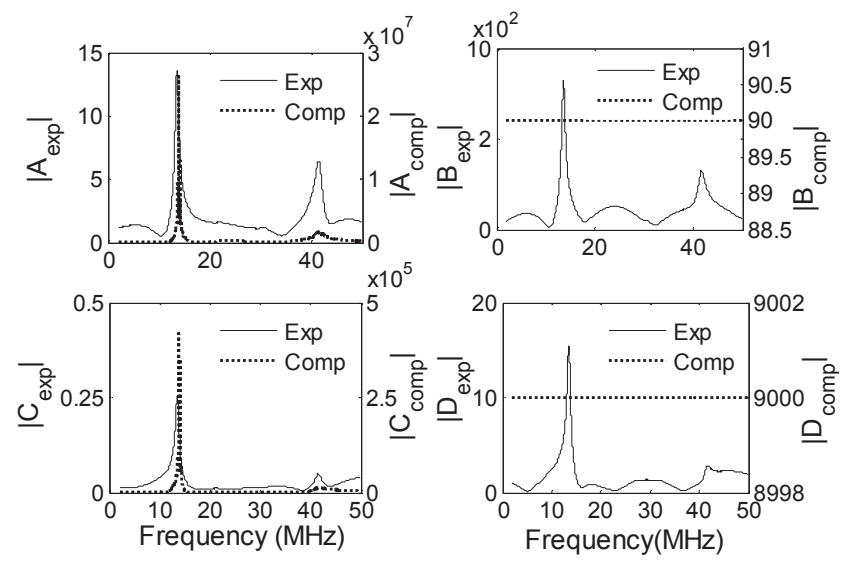

Fig. 12. A comparison of the magnitude of the experimental (Exp) and computed (Comp) values of ABCD matrices denoted by $\left(\mathrm{A}_{\text {exp }}, \mathrm{B}_{\text {exp }}, \mathrm{C}_{\text {exp }}, \mathrm{D}_{\text {exp }}\right)$ and ( $\left.\mathrm{A}_{\text {comp }}, \mathrm{B}_{\text {comp }}, \mathrm{C}_{\text {comp }}, \mathrm{D}_{\text {comp }}\right)$ respectively in the case $(1,1,2,2)$ of $P L_{1}$.

measurement. Using this procedure, alternate methods of $S_{21}$ estimations can be done as far as the notches are concerned. Use of channel estimation that is simple will surely accelerate use of PLC by detecting the notches easily for suitable mitigation.

\section{Acknowledgments}

The authors are grateful to the Department of Science and Technology, New Delhi, FIST program for providing the funds for procurement the instruments for the study.

\section{References}

[1] TIRU, B. Exploiting power line for communication purpose: Features and prospects of power line communication. In Intelligent Applications for Heterogeneous System Modeling and Design. 2015, p. 320-334. DOI: 10.4018/978-1-4666-8493-5.ch014

[2] ARtale, G., CAtaliotti, A., Cosentino, V., et al. A new low cost power line communication solution for smart grid monitoring and management. IEEE Instrumentation \& Measurement Magazine, 2018, vol. 21, no. 2, p. 29-33. DOI: 10.1109/MIM.2018.8327976

[3] MLYNEK, P., HASIRCI, Z., MISUREC, J., et al. Analysis of channel transfer functions in power line communication system for smart metering and home area network. Advances in Electrical and Computer Engineering, 2016, vol. 16, no. 4, p. 51-56. DOI: 10.4316/AECE.2016.04008

[4] Versolatto, F., TONEllo, A. M. PlC channel characterization up to $300 \mathrm{MHz}$ : Frequency response and line impedance. In Proceedings of the IEEE Global Communications Conference (GLOBECOM). Anaheim (CA, USA), 2012, p. 3525-3530. DOI: 10.1109/GLOCOM.2012.6503661

[5] TIRU, B., BAISHYA, B., SARMA, U. An analysis of indoor power line network as a communication medium using $\mathrm{ABCD}$ matrices effect of loads on the transfer function of power line. Lecture Notes in Electrical Engineering, Advances in Communication and Computing, Springer, 2015, vol. 347, p. 171-181. DOI: $10.1007 / 978-81-322-2464-8 \_14$ 
[6] MLYNEK, P., MISUREC, J., KOUTNY, M. Random channel generator for indoor power line communication. Measurement Science Review, 2013, vol. 13, no. 4, p. 206-213. DOI: 10.2478/msr-2013-0032

[7] CANETE, F. J. C., CORTES, J. A. S., DIEZ, L., et al. A channel model proposal for indoor power line communication. IEEE Communications Magazine, 2011, vol. 49, no. 12, p. 166-174. DOI: 10.1109/MCOM.2011.6094022

[8] KHALIL, K. Contributions to Indoor Broadband Power Line Communication: Channel Modeling and Data Rate Optimization. PhD Dissertation. 2015, Université de Valenciennes et du Hainaut-Cambresis. Available at: https://tel.archivesouvertes.fr/tel-01343257/document

[9] ZIMMERMANN, M., DOSTERT, K. A multipath model for the powerline channel. IEEE Transactions on Communications, 2002 vol. 50, no. 4, p. 553-559. DOI: 10.1109/26.996069

[10] PASSERINI, F., TONELLO, A. M. Power line fault detection and localization using high frequency impedance measurement. In Proceedings of the IEEE International Symposium on Power Line Communications and its Applications (ISPLC). Madrid (Spain), 2017, p. 1-5. DOI: 10.1109/ISPLC.2017.7897102

[11] MARROCCO, G., STATOVCI, D., TRAUTMANN, S. A PLC broadband channel simulator for indoor communications. In Proceedings of the IEEE 17th International Symposium on Power Line Communications and Its Applications. Johannesburg (South Africa), 2013, p. 321-326. DOI: 10.1109/ISPLC.2013.6525871

[12] RASOOL, B., RASOOL, A., KHAN, I. Impedance characterization of power line communication networks. Arabian Journal for Science and Engineering, 2014, vol. 39, p. 6255-6267. DOI: $10.1007 / \mathrm{s} 13369-014-1235-\mathrm{Z}$

[13] ANDARI, M. K., BEHESHTI, A. A. Pilot based channel estimation in broadband power line communication networks. Communications and Network, 2012, vol. 4 no. 3, p. 240-247. DOI: $10.4236 / \mathrm{cn} .2012 .43028$

[14] GALLI, S., BANWELL, T. A novel approach to the modeling of the indoor power line channel-Part II: Transfer function and its properties. IEEE Transactions on Power Delivery, 2005, vol. 20, no. 3, p. 1869-1878. DOI: 10.1109/TPWRD.2005.848732

[15] BAISHYA, R., TIRU, B., SARMA, U. An alternate method for prediction and analysis of notch characteristics in indoor power lines under varied channel conditions. Arabian Journal for Science and Engineering, 2020, vol. 45, p. 1531-1552. DOI: 10.1007/s13369-019-04052-w

[16] TIRU, B. A novel method of computation of the transfer function of unknown networks for indoor power line communication. In Proceedings of the IEEE Symposium of Computational Intelligence for Communication Networks (CIComms). Orlando (FL, USA), 2014, p. 1-7. DOI: 10.1109/CICommS.2014.7014640

[17] LINDQVIST, F. Estimation and Detection of Transmission Line Characteristics in Copper Access Network. PhD Dissertation. 2011, Lund University. Available at: https://www.semanticscholar.org/paper/Estimation-and-detectionof-transmission-line-inLindqvist/b1b222be230e2a $9 \mathrm{ab} 0 \mathrm{f5c} 82 \mathrm{~d} 0 \mathrm{fb} 8 \mathrm{f} 1 \mathrm{bfcf9c} 21 \mathrm{c} 3$

[18] MENG, H., CHEN, S., GUAN, Y. L., et al. Modeling of transfer characteristics for the broadband power line communication channel. IEEE Transactions on Power Delivery, 2004, vol. 19, no. 3, p. 1057-1064. DOI: 10.1109/TPWRD.2004.824430
[19] SASTRY, S. S. Introductory Methods of Numerical Analysis. $5^{\text {th }}$ ed. New Delhi (India): PHI Learning Limited, 2012. ISBN: 978$81-203-4592-8$

[20] CASPERS, F. RF Engineering Basic Concepts: S-parameters. 2012, p. 1-27. arXiv:1201.2346 [physics.acc-ph]

[21] FRICKEY, D. A. Conversions between S, Z, Y, h, ABCD and T parameters which are valid for complex source and load impedances. IEEE Transactions on Microwave Theory and Techniques, 1994, vol. 42, no. 2, p. 205-211. DOI: $10.1109 / 22.275248$

[22] KING, A. P., ECKERSLEY, R. J. Statistics for Biomedical Engineers and Scientists. Academic Press, 2019. ISBN: 978-0-08102939-8. DOI: 10.1016/C2018-0-02241-0

\section{About the Authors ...}

Sujit CHATTERJEE has done M.Sc. in Physics from Gauhati University, India in the year 2014 with Electronics and Condensed Matter Physics (CMP) as elective. He is pursuing $\mathrm{Ph} . \mathrm{D}$. in the topic hybrid communication techniques involving power line and visible light in the same department. He got a best presenter award in the Physics Academy of the North East (PANE) held at Diphu, India and in Research Conclave held at Guwahati, 2018.

Banty TIRU was born in Assam, India. She received her M.Sc. in Physics from Gauhati University, India with Electronics as her elective paper. She received her Ph.D. from the same university in the topic of Power Line Communication (PLC) in 2011. Dr. Tiru had been a faculty member in the Physics Department from the year 1999. Her research interests include communications, digital signal processing and atmospheric physics. At present she is working on developing hybrid communication techniques, designing of ground antennas, System on Chip applications and on clear air turbulence. She has published a number of journal and conference papers. She was one of the General Chairs of the International Conference on Advances in Electrical, Electronic and System Engineering (Guwahati, 2019). She was also a co-director of the International Centre for Theoretical Physics (ICTP), Trieste, Italy School on 'Fully Programmable Systems on Chip for Scientific Instrumentation' held at Gauhati University (2019). She is doing collaborative research with National Atmospheric Research Laboratory, India.

Rubi BAISHYA has done M.Sc. in Physics from Gauhati University in the year 2012 with Electronics and CMP as elective paper. She has completed a Ph.D. in 2020 in the topic of developing a sensor network for indoor monitoring of weather parameters using indoor power line. She has a number of conference and journal publication and got the best presenter award from the PANE conference held at Arunachal Pradesh, India in the year 2014. 